\title{
(6) OPEN ACCESS \\ Risk for lower intestinal perforations in patients with rheumatoid arthritis treated with tocilizumab in comparison to treatment with other biologic or conventional synthetic DMARDs
}

\author{
A Strangfeld ${ }_{1}^{1}$ A Richter, ${ }_{1}^{1}$ B Siegmund, ${ }^{2}$ P Herzer, ${ }^{3} \mathrm{~K}$ Rockwitz, ${ }_{1}^{4}$ W Demary, ${ }_{1}{ }^{5}$ \\ M Aringer, ${ }^{6} \mathrm{Y}^{\mathrm{A}}$ Meißner, ${ }^{1}$ A Zink, ${ }^{1,2} \mathrm{~J}$ Listing $^{1}$
}

Handling editor Tore K Kvien

- Additional material is

published online only. To view please visit the journal online (http://dx.doi.org/10.1136/an nrheumdis-2016-209773)

'Department of Epidemiology, German Rheumatism Research Centre, Berlin, Germany

${ }^{2}$ Centrum Innere Medizin mit Gastroenterologie und Nephrologie CC 13, Charité University Medicine Berlin,

Berlin, Germany

${ }^{3}$ Scientific Advisory Board,

Munich, Germany

${ }^{4}$ Goslar, Germany

${ }^{5}$ Hildesheim, Germany

${ }^{6}$ Department of Internal Medicine III, Division of Rheumatology, University Medicine Dresden, Dresden, Germany

Correspondence to Dr Anja Strangfeld, Deutsches Rheuma-Forschungszentrum Berlin, Ein Leibniz Institut, Programmbereich Epidemiologie, Charitéplatz 1, Berlin 10117, Germany; strangfeld@drfz.de

Received 22 April 2016 Revised 20 May 2016 Accepted 19 June 2016 Published Online First 12 July 2016

\section{ABSTRACT}

Objective To investigate the risk of developing lower intestinal perforations (LIPS) in patients with rheumatoid arthritis (RA) treated with tocilizumab (TCZ).

Methods In 13310 patients with RA observed in the German biologics register Rheumatoid Arthritis:

Observation of Biologic Therapy, 141 serious gastrointestinal events possibly associated with perforations were reported until 31 October 2015. All events were validated independently by two physicians, blinded for treatment exposure.

Results 37 LIPs (32 in the colon/sigma) were observed in 53972 patient years (PYs). Only two patients had a history of diverticulitis (one in TCZ). Age, current/ cumulative glucocorticoids and non-steroidal antiinflammatory drugs were significantly associated with the risk of LIP. The crude incidence rate of LIP was significantly increased in TCZ (2.7/1000 PYs) as compared with all other treatments (0.2-0.6/1000 PYs). The adjusted HR (ref: conventional synthetic (cs) diseasemodifying anti-rheumatic drugs (DMARDs)) in TCZ was $4.48(95 \% \mathrm{Cl} 2.0$ to 10.0$)$, in tumour necrosis factor- $\alpha$ inhibitor (TNFi) 1.04 (0.5 to 2.3) and in other biologic DMARDs 0.33 (0.1 to 1.4). 4/11 patients treated with TCZ presented without typical symptoms of LIP (acute abdomen, severe pain). Only one patient had highly elevated $C$ reactive protein (CRP). One quarter of patients died within 30 days after LIP (9/37), 5/11 under TCZ, 2/13 under TNFi and 2/11 under csDMARD treatment.

Conclusions The incidence rates of LIP under TCZ found in this real world study are in line with those seen in randomised controlled trials of TCZ and higher than in all other DMARD treatments. To ensure safe use of TCZ in daily practice, physicians and patients should be aware that, under TCZ, LIP may occur with mild symptoms only and without CRP elevation.

\section{INTRODUCTION}

Lower intestinal perforations (LIPs) are rare in the CrossMark

To cite: Strangfeld A, Richter A, Siegmund B, et al. Ann Rheum Dis 2017;76:504510 .
In patients with rheumatoid arthritis (RA), perforations of the gastrointestinal (GI) tract have been a concern for quite a long time. In the prebiologic era, GI complications were among the most common causes of death in patients with RA. ${ }^{4}$ At that time, concerns mainly referred to perforations of the upper GI tract, for which non-steroidal antiinflammatory drugs (NSAIDs) and glucocorticoids (GCs) are the most important risk factors. ${ }^{5}$ However, in addition to risks for the upper GI tract, for the lower GI tract a higher risk for diverticular complications was found on NSAIDs in several case-control studies with ORs ranging from 1.8 to $11.2 .^{6-8}$

Similarly, GCs were found to be strongly associated with lower GI perforations ${ }^{9-11}$ with HRs in patients with RA of 2.8 (95\% CI 1.3 to 6.1) compared with non-users ${ }^{9}$ or 4.7 (95\% CI 1.9 to 12.0 ) when GCs and NSAIDs were used concomitantly.

Since the approval of the first biologics, the incidence of GI tract complications in RA was expected to decrease with the decreasing use of NSAIDs and high-dose GCs. However, this is only the case if concomitant GCs can be reduced by effective therapy. Curtis et al investigated the risk for GI tract complications on tumour necrosis factor- $\alpha$ inhibitors (TNFi) and found a higher incidence of hospitalised GI perforation with concomitant GCs (1.12 (95\% CI 0.5 to 2.5$) / 1000$ patient years (PYs)) than without $(0.47$ (95\% CI 0.2 to 0.98$)) .^{9}$ The British Biologics Register (BSRBR) ${ }^{12}$ compared the risk of GI perforations in patients treated with TNFi and those on conventional synthetic (cs) disease-modifying anti-rheumatic drugs (DMARDs) only. While there was no statistically important elevation of the risk associated with TNFi with an incidence rate of lower GI perforations of 0.39 / 1000 PYs, concomitant GCs were the most important risk factor, conveying a 2.9 (95\% CI 1.5 to 5.3$)$ times higher risk, confined to lower GI perforations with a HR of 8.0 (95\% CI 2.6 to 24.1).

The clinical development programme of tocilizumab (TCZ) for the treatment of RA identified GI perforations as important risk. An integrated safety analysis of eight trials and long-term extension studies with TCZ reported that no GI perforation had occurred in the group treated with csDMARDs only, but 26 such cases were identified in patients of the group ever exposed to TCZ, resulting in an 
incidence rate of 2.8/1000 PYs. ${ }^{13}$ Eighteen of these perforations occurred in the colon. Due to the early escape design of the trials, only 825 PYs were available in the csDMARD group, limiting the significance of the finding.

Since there is little information on the overall incidence of LIP in patients with RA exposed to specific treatments, no robust comparisons of the incidence rate of GI perforations could be made between TCZ-treated patients and those on other therapies. Several other factors, such as diverticular disease, high disease activity or long-standing high-dose GC or NSAID use, could also have accounted for the higher incidence in TCZ-treated patients. Further, the few studies existing in this field differ considerably in study design, case definition and population studied, the latter impacting on the background risk.

Prospective observational cohort studies, such as the German biologics register Rheumatoid Arthritis Observation of Biologic Therapy (RABBIT), have the advantage that all patients who start treatment with one of the approved biologic agents are eligible to be enrolled. In addition, a control group treated with csDMARDs only is observed under the same protocol. This design enables studying differences in incidence rates of adverse events occurring under different treatments. The aim of this study was to identify risk factors for LIP within the RABBIT register, taking concomitant (time varying) dosages of NSAIDs and GCs into account. We thereby aimed to examine the clinical signs and symptoms of LIP events to inform treating physicians how to advise their patients when starting a new treatment.

\section{METHODS}

\section{Patients}

We used data of the German biologics register RABBIT captured and validated until 31 October 2015.

RABBIT is an ongoing observational cohort study that started in May 2001. Since then, patients with RA are enrolled when starting a treatment with a biologic DMARD (bDMARD), or csDMARD after failure of at least one csDMARD. Once enrolled, patients are observed for at least 5 years regardless of treatment terminations and changes (with the option to extend observation for another 5 years, if the patient agrees).

The study protocol was approved in 2001 by the ethics committee of the Charite University School of Medicine, Berlin. Each patient participating in the study gave written informed consent before study entry.

\section{Assessments and procedures}

During follow-up, information from rheumatologists and patients is captured at regular intervals: at baseline, after 3 and 6 months, and thereafter every 6 months. Data collected include clinical status, disease activity (including disease activity score based on 28 joints (DAS28)), details on DMARD treatment (substance, dosage, application, start and stop dates, reasons for stopping) and concomitant treatments like NSAIDs (yes/no) and GCs (actual dose and mean dose since last questionnaire). At every time point of follow-up, all adverse events that occurred since last questionnaire are reported. At baseline and every 2 years, rheumatologists report comorbid conditions in predefined groups and in plain text. Patients report, among other items, physical function (using the Hannover Functional Status Questionnaire, $\mathrm{FFbH}$ ).

Adherence to scheduled visits is monitored closely. Investigations of dropouts (defined as two missing follow-ups) are performed regularly. This includes inquiries to local administration offices regarding patient's vital status and, if the patient had died, obtaining the causes of death from the health authorities. Complete details have been published. ${ }^{14}$

\section{Outcomes}

\section{Primary outcome}

Incidence of LIPs in patients exposed to TCZ, csDMARDs, TNFi, abatacept or rituximab.

\section{Secondary outcomes}

Clinical signs and symptoms of LIPs in these patients.

Thirty-day mortality after LIPs.

\section{Outcome validation}

To capture all LIPs, we first identified all events that might be associated with GI perforations (including also haemorrhages and bleedings of the intestinal tract) via a predefined group of MedDRA terms. These included all events of the standard MedDRA query (SMQ) 'GI perforation' plus 51 additional MedDRA codes (see online supplementary table S1). Based on medical records and specific queries to the treating rheumatologists, all events were validated first by the leading physician of RABBIT (AS). An additional external validation was done by the Head of the Department for Gastroenterology of the Charite University Medicine (BS). At time of validation, both reviewers were blinded for the patient's treatment exposure. Only events with a definite, non-iatrogenic and non-traumatic LIP were selected for the analysis. We only counted perforations localised below the duodenojejunal junction (ligament of Treitz) as lower GI perforation. The topmost localised perforation included in the analysis was in the terminal ileum.

\section{Definition of DMARD exposure}

Treatment was assigned using a risk window for exposure to bDMARDs: a patient was considered exposed to a certain bDMARD at the time of the event if he/she had received at least one dose of the drug within 3 months (rituximab 9 months) prior to the event. Patients unexposed to bDMARDs in this risk window were assigned to the csDMARD group.

All substances targeting TNF (adalimumab, certolizumab pegol, etanercept, golimumab, infliximab) were subsumed under TNFi since we had not seen any differences in incidence rates.

\section{Statistical analysis}

Multiple Cox regression was applied to compare the risks of LIPs between the treatment groups. Adjustment was made for age, sex, treatment with GCs and NSAIDs. We distinguished current (Cox regression 1) and cumulative (Cox regression 2) treatment with NSAIDs and GCs. For each patient, the portion of visits with new or ongoing NSAIDs treatment was used as a proxy for NSAID use (range: 0 to 1). Regarding cumulative treatment with GCs, we proceeded in a similar way, but considered each month with a dose of $>5-10 \mathrm{mg} /$ day with a weight of 0.5 and each month with a dose of $>10 \mathrm{mg} /$ day with a weight of 1. Further covariables (body mass index (BMI), number of bDMARD failures, DAS28) were additionally investigated in univariate Cox regression. We applied two sensitivity analyses: (a) we restricted the cohort to a subgroup of patients recruited after 2009 and (b) we repeated Cox regression 1 and 2 but restricted the reference group to biologic naïve patients. Exact Poisson and Clopper-Pearson binomial CIs were calculated for incidence rates and proportions. The uncertainty of HRs is shown in $95 \%$ CIs. 


\section{RESULTS}

\section{Patients}

Until 31 October 2015, 13310 patients had been enrolled in the RABBIT register. Patients in the csDMARD-treated biologic naïve control group were older and had shorter disease duration at inclusion than TNFi-treated patients. Patients starting treatment with non-TNFi biologics had the longest disease duration and the highest number of prior treatment failures (table 1). Most of them (66.1\%) had prior TNFi treatment.

The prevalence of chronic GI disease at baseline was lowest in the csDMARD-treated group and highest in patients starting treatment with abatacept. Chronic diverticulosis was reported in 33 patients. In two patients, a history of diverticulitis was known to the rheumatologist, one of these patients had a perforated diverticulum before inclusion in the register. None of the patients with chronic GI disease at baseline developed an intestinal perforation during follow-up.

\section{Incidence of GI perforations}

In 53972 PYs of follow-up, 141 adverse events possibly describing GI perforations were reported. After internal and external medical review, 44 GI perforations were identified (figure 1). Seven were localised in the upper GI tract: three under TNFi resulting in an incidence rate of 0.12 (95\% CI 0.02 to 0.35 )/ $1000 \mathrm{PYs}$ and four under csDMARD $(0.22$ (95\% CI 0.06 to 0.57)/1000 PYs).

Thirty-seven GI perforations were localised in the lower GI tract: 32 in the colon/sigma, 4 in the appendix, and 1 in the terminal ileum. Similar incidence rates were observed for patients exposed to csDMARDs (0.6 (95\% CI 0.3 to 1.1$) / 1000$ PYs), TNFi $(0.5$ (95\% CI 0.3 to 0.9$) / 1000$ PYs), abatacept $(0.5$ (95\% CI 0.01 to 2.8$) / 1000 \mathrm{PYs})$ and rituximab $(0.2$ (95\% CI 0.01 to 1.1)/1000 PYs), whereas the incidence rate for patients exposed to TCZ was significantly higher (2.7 (95\% CI 1.4 to 4.8$)$ / 1000 PYs) (figure 2). These crude incidence rates correspond to numbers needed to harm of 1647,1911 and 371 in patients treated with csDMARDs, TNFi and TCZ, respectively. Sensitivity analysis (a) showed similar rates (see online supplementary figure $\mathrm{S} 1$ ).

\section{Univariate and multivariate risk evaluation of LIP}

Twenty-eight of the 37 patients who developed LIP had concomitant GCs, with a daily dose of $\geq 7.5 \mathrm{mg}$ in 12 patients. Compared with those treated with TCZ, the average dose of GCs was higher in patients treated with TNFi and lower in csDMARD-treated patients (table 2). Further characteristics of patients who developed perforations did not differ significantly between the various treatment groups.

In the univariate analysis higher age, treatment with TCZ, current as well as cumulative GCs, and cumulative NSAIDs but none of the other risk factors (eg, sex, disease activity (DAS28), BMI and number of previous biologics) were significantly associated with LIP. In the multivariate analysis, higher age, current and cumulative use of GCs and NSAIDs were significantly associated with a higher risk of LIP, in addition to treatment with TCZ (table 3). Compared with csDMARDs, exposure to TCZ was associated with a 4.5 times higher risk for LIP (95\% CI 2.01 to 9.99) (table 3, Cox regression 2), whereas no association was found for TNFi, abatacept or

\begin{tabular}{|c|c|c|c|c|c|}
\hline Parameter & csDMARDs & TNFi & TCZ & $A B A$ & RTX \\
\hline $\mathrm{N}$ & 4423 & 6711 & 877 & 371 & 928 \\
\hline Patient years, sum & 18113 & 24851 & 4082 & 1976 & 4950 \\
\hline Age, years, mean (SD) & $57.6(12.3)$ & $54.9(12.6)$ & $56.7(12.8)$ & $58.1(12.9)$ & $58.7(12.1)$ \\
\hline Female & $3345(75.6)$ & $5113(76.2)$ & $685(78.1)$ & $282(76.0)$ & $719(77.5)$ \\
\hline Rheumatoid factor positive & $2763(62.6)$ & $4983(74.9)$ & $608(72.2)$ & $267(74.2)$ & $764(82.9)$ \\
\hline No. of previous csDMARDs, mean (SD) & $1.4(0.9)$ & $2.7(1.4)$ & $2.2(1.1)$ & $2.4(1.3)$ & $2.6(1.2)$ \\
\hline No. of previous bDMARDs, mean (SD) & $0(0.2)$ & $0.2(0.6)$ & $1.0(1.1)$ & $1.4(1.3)$ & $1.4(1.1)$ \\
\hline NSAIDs & $1497(33.8)$ & $2695(40.2)$ & $293(33.4)$ & $151(40.7)$ & $351(37.8)$ \\
\hline Glucocorticoids, not available & $9(0.2)$ & $18(0.3)$ & 0 & 0 & $9(1.0)$ \\
\hline Glucocorticoids, $<5$ mg/day & $1412(31.9)$ & $1682(25.1)$ & $240(27.4)$ & $80(21.6)$ & $215(23.2)$ \\
\hline Glucocorticoids, 5-10 mg/day & $2057(46.5)$ & $3058(45.6)$ & $389(44.4)$ & $182(49.1)$ & $386(41.6)$ \\
\hline Glucocorticoids, $\geq 10 \mathrm{mg} /$ day & $945(21.4)$ & $1953(29.1)$ & $248(28.3)$ & $109(29.4)$ & 318 (34.3) \\
\hline Disease duration, years, mean (SD) & $7.2(8.0)$ & $10.7(9.2)$ & $10.6(8.7)$ & $12.0(9.0)$ & $13.8(9.9)$ \\
\hline DAS28, mean (SD) & $4.7(1.3)$ & $5.4(1.3)$ & $5.2(1.3)$ & $5.4(1.3)$ & $5.3(1.3)$ \\
\hline CRP, mg/L, mean (SD) & $14.1(20.5)$ & $21.2(29.4)$ & $18.0(26.1)$ & $19.6(26.3)$ & $18.3(24.6)$ \\
\hline $\mathrm{FFbH}$, mean (SD) & $69.4(21.7)$ & $61.4(23.3)$ & $62.6(24.2)$ & $58.6(23.6)$ & $55.9(23.8)$ \\
\hline BMI, mean (SD) & $27.4(5.3)$ & $26.4(5.3)$ & $26.7(5.4)$ & $26.8(5.8)$ & $26.3(5.1)$ \\
\hline $\mathrm{BMI} \geq 30 \mathrm{~kg} / \mathrm{m}^{2}$ & $1167(26.4)$ & $1412(21.0)$ & $218(24.9)$ & $81(21.8)$ & $181(19.5)$ \\
\hline Diabetes mellitus & $436(9.9)$ & $668(10.0)$ & $102(11.6)$ & $46(12.4)$ & $111(12.0)$ \\
\hline Hyperlipoproteinemia & $315(7.1)$ & $557(8.3)$ & $82(9.4)$ & $44(11.9)$ & $106(11.4)$ \\
\hline Diverticulosis/prior diverticulitis & $12(0.3)$ & $11(0.2)$ & $3(0.3)$ & $4(1.1)$ & $5(0.5)$ \\
\hline Gastrointestinal diseases & 159 (3.6) & $339(5.1)$ & $43(4.9)$ & $26(7.0)$ & $59(6.4)$ \\
\hline Chronic renal disease & $102(2.3)$ & $295(4.4)$ & $49(5.6)$ & $24(6.5)$ & $61(6.6)$ \\
\hline
\end{tabular}

Values are numbers of patients (\%) unless otherwise specified.

ABA, abatacept; bDMARDs, biologic disease-modifying anti-rheumatic drugs; BMI, body mass index; CRP, C reactive protein; csDMARDs, conventional synthetic disease-modifying anti-rheumatic drugs; DAS28, disease activity score based on 28 joints; FFbH, Funktionsfragebogen Hannover (physical function in \%); NSAIDs, non-steroidal anti-inflammatory drugs; RABBIT, Rheumatoid Arthritis Observation of Biologic Therapy; RTX, rituximab; TCZ, tocilizumab; TNFi, tumour necrosis factor- $\alpha$ inhibitor 


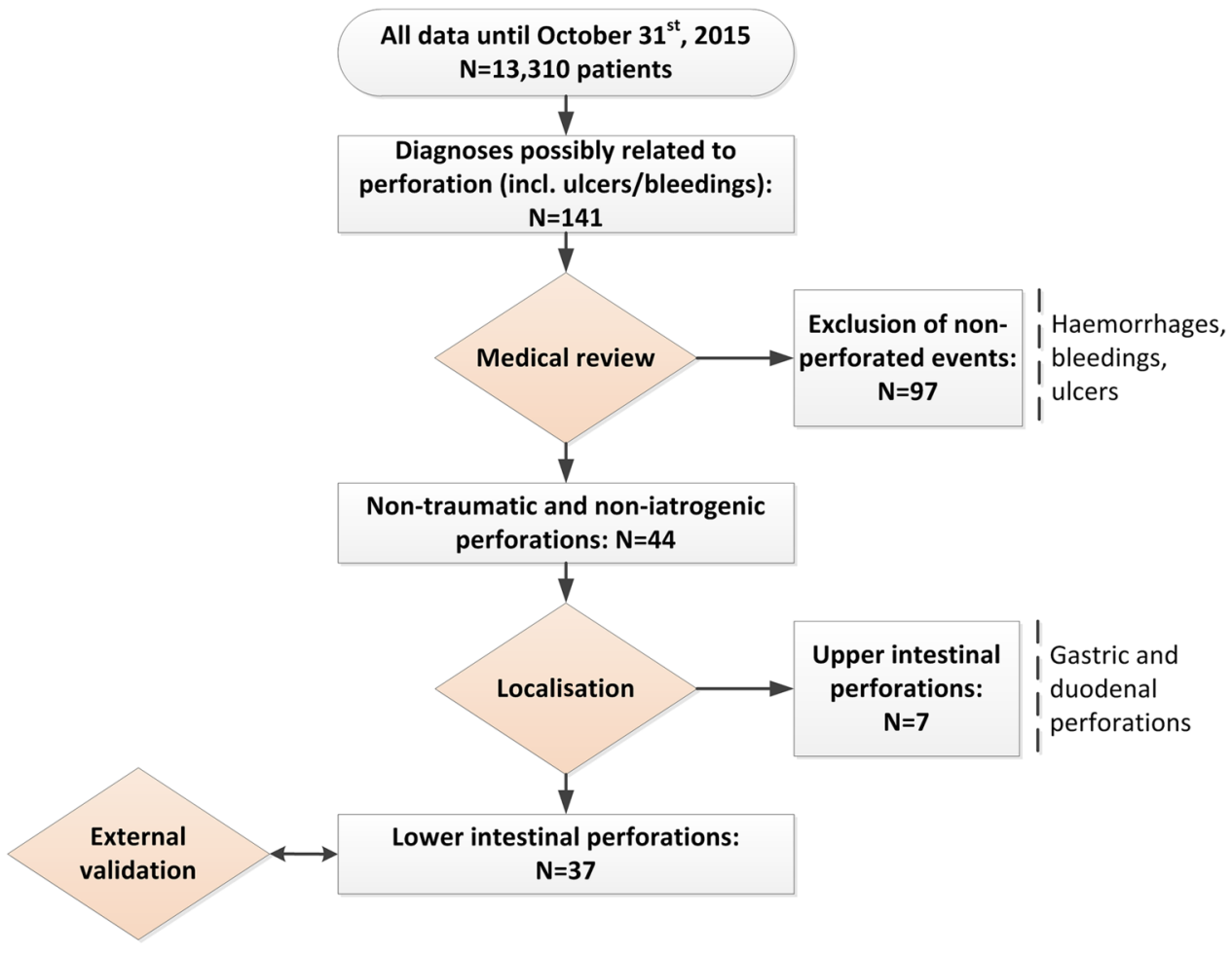

Figure 1 Flowchart of case selection and validation process.

rituximab. Sensitivity analyses (b) did not show different results (see online supplementary table S3).

\section{Clinical signs and outcome of LIPs}

While $90 \%$ of the patients with csDMARD and $60 \%$ of those on TNFi reported acute abdominal pain, this was only the case for 27\% (three cases) on TCZ. The majority of patients treated with csDMARDs or other bDMARDs had very high $\mathrm{C}$ reactive protein (CRP) values (above $100 \mathrm{mg} / \mathrm{L}$ ). In contrast, this was the case in only one patient treated with TCZ.

Online supplementary table S2 shows demographics, CRP values, features of clinical presentation and comedication with GC of each patient who developed LIP on TCZ.

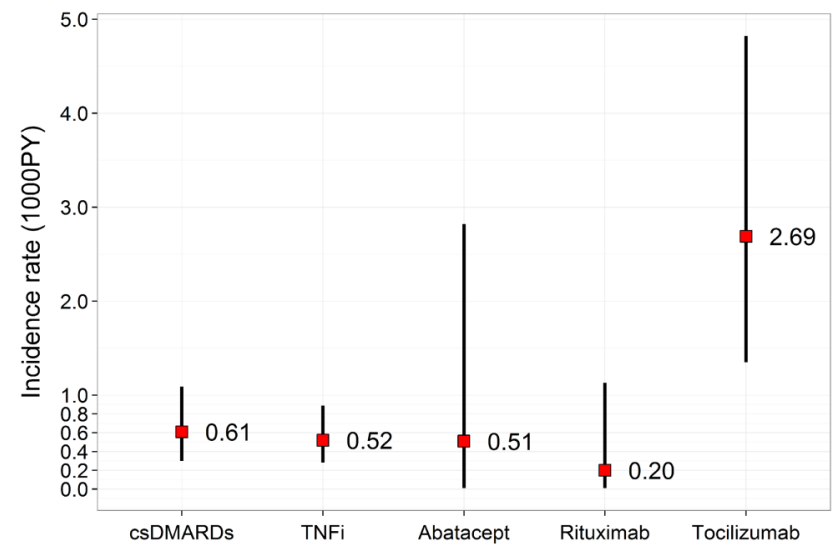

Figure 2 Incidence rates of lower intestinal perforation stratified by DMARDs. Events/patient years (PYs): csDMARDs=11/18 113;

TNFi=13/24 851 (adalimumab=6, etanercept $=6$, infliximab=1); Abatacept=1/1976; Rituximab=1/4950; Tocilizumab=11/4082. csDMARD, conventional synthetic DMARD; TNFi, tumour necrosis factor- $\alpha$.
According to classifications of colonic diseases, LIPs are considered as complicated diverticulitis (in contrast to diverticulitis without perforation). In total, 92 events of diverticulitis were reported during follow-up. The proportion of patients who developed a perforation was higher in patients treated with TCZ than in all other treatment groups (see table 4).

The 30 -day mortality after perforation was $24 \%$ in all groups (9/37 patients). The mortality in patients on TCZ (46\%) was considerably elevated, although not statistically significant $(\mathrm{p}=0.09$, Fisher's exact test) (table 4).

\section{DISCUSSION}

Our objective was to evaluate the risk for LIP under various treatments by using the data of a large German RA cohort. We observed a significantly elevated risk for LIP in patients treated with TCZ compared with patients treated with csDMARD and also as compared with patients treated with TNFi or other biologics. LIPs are uncommon events that occurred in patients exposed to TCZ with an incidence of 2.7 per 1000 PYs only, but were associated with a 30 -day mortality of $46 \%$.

In patients treated with TCZ, symptomatic diverticulitis was more often associated with perforation than in other treatments. In these patients, the clinical presentation tended to be milder than in other patients. This might explain the finding from van Vollenhoven et $a l^{15}$ that in the majority of patients in the TCZ clinical development programme who had a perforation, the diverticulitis was recognised only after the perforation had occurred.

The incidence rate of LIP in patients treated with TCZ in our cohort was comparable to that reported from randomised controlled trials with TCZ, postmarketing surveillance studies, spontaneous reports or US-based healthcare data in which per 1000 PYs 2.8, 2.4 and 2-2.3 LIPs, respectively, were observed. $^{13} 1617$ Observational cohort studies reported lower incidence rates in patients with RA not treated with TCZ: in the 
Table 2 Clinical characteristics during follow-up of patients who developed LIP

\begin{tabular}{|c|c|c|c|c|c|}
\hline Parameter & csDMARDs & TNFi & TCZ & $A B A$ & RTX \\
\hline $\mathrm{N}$ & 11 & 13 & 11 & 1 & 1 \\
\hline Age at LIP, years, mean (SD) & $66.8(4.8)$ & $67.3(8.3)$ & $69.2(7.6)$ & [73] & [61] \\
\hline Rheumatoid factor positive & $11(100)$ & $12(92.3)$ & $6(54.5)$ & [1 (100)] & [1 (100)] \\
\hline No. of previous bDMARDs, mean (SD) & $0.7(1.3)$ & $1.8(0.8)$ & $3.3(1.6)$ & [4] & [4] \\
\hline Cumulative NSAID treatment* ${ }^{\star}$, mean (SD) & $0.4(0.4)$ & $0.4(0.3)$ & $0.3(0.4)$ & [1] & {$[0.5]$} \\
\hline$\leq 6$ months, mean (SD) & $3.8(0.7)$ & $4.0(1.7)$ & $3.5(2.1)$ & [5.0] & [5.4] \\
\hline$\leq 12$ months, mean (SD) & $3.8(0.7)$ & $4.0(1.6)$ & $3.7(2.0)$ & [5.0] & {$[5.5]$} \\
\hline \multicolumn{6}{|l|}{ CRP (mg/L) prior to LIP } \\
\hline$\leq 6$ months, mean (SD) & $8.1(7.9)$ & $25.8(33.4)$ & $11.5(17.6)$ & {$[0.7]$} & [3.8] \\
\hline$\leq 12$ months, mean (SD) & $10.6(8.5)$ & $28.3(33.0)$ & $16.0(21.5)$ & {$[0.7]$} & {$[4.2]$} \\
\hline \multicolumn{6}{|l|}{ Glucocorticoids prior to LIP } \\
\hline Average over 6 months, mean (SD) & $5.6(3.8)$ & $9.7(9.3)$ & $7.5(7.5)$ & {$[5.0]$} & {$[10.0]$} \\
\hline Average over 12 months, mean (SD) & $5.6(3.4)$ & $9.6(9.0)$ & $8.1(7.3)$ & [5.0] & {$[10.0]$} \\
\hline
\end{tabular}

${ }^{*}$ Cumulative treatment with NSAID (range: 0 to 1 ) was calculated for each patient as: no. of follow-ups with concomitant NSAID use divided by the total no. of follow-ups. ABA, abatacept; bDMARDs, biologic disease-modifying anti-rheumatic drugs; CRP, C reactive protein; cSDMARDs, conventional synthetic disease-modifying anti-rheumatic drugs; DAS28, disease activity score based on 28 joints; ESR, erythrocyte sedimentation rate; LIP, lower intestinal perforation; NSAID, non-steroidal anti-inflammatory drug; RTX, rituximab; TCZ, tocilizumab; TNFi, tumour necrosis factor- $\alpha$ inhibitor.

Table 3 Univariate HRs and results of multiple Cox regression

\begin{tabular}{|c|c|c|c|c|c|c|}
\hline & \multicolumn{2}{|c|}{ Univariate } & \multicolumn{2}{|c|}{$\begin{array}{l}\text { Multiple } \\
\text { Cox regression } 1\end{array}$} & \multicolumn{2}{|c|}{$\begin{array}{l}\text { Multiple } \\
\text { Cox regression } 2\end{array}$} \\
\hline & HR & $95 \% \mathrm{Cl}$ & HR & $95 \% \mathrm{Cl}$ & HR & $95 \% \mathrm{Cl}$ \\
\hline Age at event (by 5 years) & 1.48 & (1.25 to 1.75$)$ & 1.55 & (1.30 to 1.84$)$ & 1.57 & (1.32 to 1.87 ) \\
\hline Male & 1.68 & (0.84 to 3.34$)$ & 1.58 & (0.79 to 3.20$)$ & 1.45 & (0.72 to 2.90$)$ \\
\hline BMI & 1.00 & (0.94 to 1.06$)$ & & & & \\
\hline \multicolumn{7}{|l|}{ bDMARD failures (reference: 0 ) } \\
\hline 1 bDMARD & 1.54 & (0.67 to 3.52 ) & & & & \\
\hline$\geq 2$ bDMARDs & 0.71 & (0.17 to 3.00$)$ & & & & \\
\hline DAS28 (current) & 1.14 & (0.92 to 1.42 ) & & & & \\
\hline DAS28 (average last 12 months) & 1.16 & (0.91 to 1.48 ) & & & & \\
\hline \multicolumn{7}{|l|}{ DMARD (reference: csDMARDs) } \\
\hline TNFi & 0.84 & (0.39 to 1.80$)$ & 1.00 & (0.46 to 2.20$)$ & 1.04 & (0.48 to 2.26$)$ \\
\hline Other bDMARDs & 0.40 & (0.09 to 1.78$)$ & 0.41 & (0.09 to 1.84 ) & 0.33 & (0.08 to 1.44$)$ \\
\hline Tocilizumab & 4.17 & (1.87 to 9.27$)$ & 5.11 & (2.31 to 11.3 ) & 4.48 & (2.01 to 9.99 ) \\
\hline \multicolumn{7}{|l|}{ GCs } \\
\hline Current GC (by 5 mg) & 1.22 & (1.13 to 1.31$)$ & 1.28 & (1.18 to 1.38$)$ & & \\
\hline Cumulative GCs* & 1.81 & (1.47 to 2.22 ) & & & 1.87 & (1.50 to 2.33 ) \\
\hline \multicolumn{7}{|l|}{ NSAIDs } \\
\hline Current NSAID & 1.80 & (0.92 to 3.53$)$ & 2.18 & (1.11 to 4.31$)$ & & \\
\hline Cumulative NSAIDs† & 2.71 & (1.20 to 6.12$)$ & & & 3.00 & (1.33 to 6.82 ) \\
\hline
\end{tabular}

In Cox regression 1, we adjusted for current doses of GCs and NSAIDs, whereas in Cox regression 2 for cumulative doses.

${ }^{*}$ Concomitant GC use (range: 0 to 1) was calculated for each patient as the area under curve of follow-up month with medium doses ( $>5$ to 10 mg/day, weight of 0.5 ) plus follow-up month with high doses (>10 mg/day, weight of 1 ) and then divided by total no. of follow-up months.

tCumulative treatment with NSAIDs (range: 0 to 1 ) was calculated for each patient as: no. of follow-ups with concomitant NSAIDs use divided by the total no. of follow-ups. bDMARD, biologic disease-modifying anti-rheumatic drug; BMI, body mass index; csDMARDs, conventional synthetic disease-modifying anti-rheumatic drugs; DAS28, disease activity score based on 28 joints; GC, glucocorticoid; NSAIDs, non-steroidal anti-inflammatory drugs; other bDMARDs, abatacept and rituximab; TNFi, tumour necrosis factor- $\alpha$ inhibitor.

Rochester cohort, a rate of $1.3 / 1000 \mathrm{PYs}^{18}$ in 813 patients diagnosed with RA between 1980 to 2008 (and followed until 2009) was observed. Only $17 \%$ of the patients had ever been exposed to bDMARDs and $77 \%$ had concomitant GCs. From
US claims data, ${ }^{19}$ a rate of $0.87 / 1000$ PYs in patients with RA was reported. In the British Biologics Register, an incidence of LIP of $0.39 / 1000$ PYs in patients on TNFi treatment and of $0.15 / 1000$ PYs in biologic naïve csDMARD-treated patients was 
Table 4 Incident diverticulitis, perforations and lethal perforations

\begin{tabular}{|c|c|c|c|c|}
\hline & \multirow[b]{2}{*}{ PYs } & \multicolumn{3}{|l|}{ Total no. of diverticulitis } \\
\hline & & $\begin{array}{l}\text { (Incidence rate/1000 PYs } \\
(95 \% \mathrm{Cl}) \text { ) }\end{array}$ & $\begin{array}{l}\text { Thereof: no. of LIP } \\
\text { (proportion, }\left(95 \% \mathrm{Cl}^{*}\right) \text { ) }\end{array}$ & $\begin{array}{l}\text { No. of patients died within } 30 \text { days } \\
\text { after LIP (proportion, }\left(95 \% \mathrm{Cl}^{*}\right) \text { ) }\end{array}$ \\
\hline csDMARDs & 18113 & $34(1.9(1.3$ to 2.6$))$ & $11(32.4$ (17.4 to 50.5$))$ & $1(9.1(0.0$ to 41.3$))$ \\
\hline Tocilizumab & 4082 & $16(3.9(2.2$ to 6.4$))$ & $11(68.7$ (41.3 to 89.0$))$ & 5 (45.5 (16.8 to 76.6$))$ \\
\hline Abatacept & 1976 & $1(0.5(0.0$ to 2.8$))$ & $1(100(2.5$ to 100$))$ & $0(0(0$ to 97.5$))$ \\
\hline
\end{tabular}

*Exact Clopper-Pearson 95\% Cl for the proportion.

csDMARDs, conventional synthetic disease-modifying anti-rheumatic drugs; IR, incidence rate per 1000 PYs; LIP, lower intestinal perforations; PYs, patient years; TNFi, tumour necrosis factor- $\alpha$ inhibitor.

found. ${ }^{12}$ GCs were the most important risk factor for LIP in this study.

Comparable to other studies, we also observed a higher risk for patients who were older, male or treated with GCs in higher dosages or NSAIDs in higher frequency. Minor differences in the distribution of these risk factors between the treatment groups did, however, not explain the higher incidence of LIPs in TCZ-treated patients. Adjustment for these factors did not decrease the HR for developing a LIP under TCZ.

Biological mechanisms may also support the increased risk of LIP in patients treated with TCZ: the interleukin 6 (IL-6) receptor targeted by TCZ seems to have an important function of the intestinal barrier. It is hypothesised that locally accumulated fat tissue may cover inflamed diverticula, similar to creeping fat in Crohn's disease which covers inflamed intestinal segments and where IL-6 is predominantly found. ${ }^{20}{ }^{21}$ Creeping fat may limit the transmural intestinal inflammation to the intestine. ${ }^{22} 23$

Our study has strengths and limitations. An important risk factor of LIP is previous diverticulitis that was likely underreported at enrolment into RABBIT. Adjustment for this risk factor was not possible since none of the patients with a history of diverticulitis, diverticulosis or another chronic GI disease developed a LIP. However, patients with prior diverticulitis are unlikely to be overrepresented in patients treated with TCZ since first clinical trials of TCZ reported a higher risk of LIP and the German Society for Rheumatology recommended not using TCZ in patients with a history of diverticulitis (http:// dgrh.de/rheumatocilizumab.html). This may have caused a lower reporting threshold for LIP under TCZ treatment. Nevertheless, we do not assume different reporting behaviour for LIPs due to the severity of the events requiring hospitalisation and possibly leading to severe sequelae. An underreporting of LIPs is therefore not likely under any treatment. The rather constant reporting rates over time support this assumption. In addition, all LIPs were diagnosed by treating gastroenterologists or surgeons of general hospitals and not the rheumatologists participating in RABBIT.

The low numbers of LIPs observed in the register were another limitation which restricted the number of covariables in the Cox regression to adjust for confounding by indication. In addition, the risk conveyed by cumulative NSAID use could only be considered by a proxy since exact doses and start/stop dates of NSAIDs as well as the exposure prior to enrolment in RABBIT are not comprised in our data. The effect of long-term NSAID use in high doses might therefore be inadequately estimated.

The strengths of our study are the prospective design, the comprehensive case validation with independent external validation, the availability of clinical information on the course and outcome of LIP and the long-term follow-up of patients exposed to different treatments including treatment switches which allows comparative analyses.

\section{CONCLUSION}

This is the first comparative analysis of real-life data on the risk of LIP, covering all DMARDs available in Germany for the treatment of RA. In agreement with the results from the TCZ clinical development programme which qualified LIP as important identified risk, we found a rate of 2.7/1000 PYs in patients treated with TCZ. This rate was significantly higher than in other biologic agents or csDMARDs. It is of clinical importance that the majority of patients who experienced a LIP on TCZ did not have a history of diverticulitis. Further, some patients with LIP presented with relatively mild symptoms. In combination with the suppressed values of CRP under TCZ treatment, this may lead to a delayed diagnosis by non-specialised physicians not familiar with TCZ. Rheumatologists should be aware that IL-6 inhibition can be associated with an increased risk of LIP in patients with prior diverticulitis. Patients should be advised to observe signs and symptoms of LIP carefully and to inform nonspecialised doctors that CRP, in their case, cannot be interpreted as a marker of diverticular inflammation. This could contribute to reduce a rare but serious risk in daily care.

Acknowledgements The authors thank all participating rheumatologists for their support of RABBIT. Particularly: Klopsch T, Krause A, Liebhaber A, Richter C, Bergerhausen $H$, Tony $H$, Dockhorn $R$, Eisterhues $C$, Bussmann $A$, von Hinuber $U$, Grasler A, Kapelle A, Kekow J, Wassenberg S, Wilden E, Ochs W, Burmester G, Zinke S, Krummel-Lorenz B, Edelmann E, Remstedt S, Meier L, Bohl-Buhler M, Kellner H, Berger S, Balzer S, Pick D, Stille C, Tremel H, Lebender S, Kruger K, Korber H, Stander E, Thiele A, Karberg K, Wiesmuller G, Weis K, Kuhne C, Schwarze I, Burmester G, Backhaus M, Zanker M, Herzer P, Sorensen H, Fricke-Wagner H, Schulze-Koops H, Grunke M, Harmuth W, Spathling-Mestekemper S, Dexel T, Alliger K, Moosig F, Schneider M, Schuch F, Wendler J, Kleinert S, Grebe T, Manger K, Karger T, Menne H, Fliedner G, Gauler G, Walter J, Reck A, Hamann F, Krause D, Hantsch J, Rech J, Seifert A, Schmitt-Haendle M, Biewer W, Leumann K, Prothmann U, Mobius C, Gause A, Eidner T, Euler H, Wiesent F, Fischer K, Petersen V, Riechers E, Alten R, Mobius E, Mark S, Rump J, Sekura M, Teipel A, Dahmen G, Jahn A, Kittel G, Hauser M, Donath G, Henes J, Zeh G, Schroder J, Hohle M, Haux R, Zeidler $G$, Jendro M, Liman W.

Contributors $A S, A R$, JL and $A Z$ had full access to all data of this study and take responsibility for data integrity and accuracy of the analysis. JL, AZ and AS: study concept and design. PH, KR, WD, MA, YM and BS: acquisition of the data. BS: external validation of diagnoses. AR, JL, AZ and AS: analysis and interpretation of the data.

AS and YM: drafting the manuscript. $A S, A R, B S, P H, K R, W D, M A, Y M, A Z$ and JL: critical revision of the manuscript for important intellectual content.

Funding The German Biologics Register RABBIT is supported by a joint, unconditional grant from AbbVie, Bristol-Myers Squibb, Celltrion, Hospira, MSD 
Sharp \& Dohme, Pfizer, Roche and UCB. The principal investigators and their team had full academic freedom in study design and conduct, data analysis and publication of results. These stipulations were delineated in their contract with the sponsors. For the purpose of information, all six funding companies received the manuscript 30 days prior to submission. Publication of this article was not contingent on their approval. The data interpretation, drafting, critical revision and approval of the final manuscript were performed solely by the authors.

Competing interests AS: personal fees from BMS, MSD, Pfizer, Roche and Sanofi-Aventis outside the submitted work. AR: honoraria from Pfizer. BS: consultant for Abbvie, Janssen, Hospira, MSD, Mundipharma and Takeda and speaker fees from Abbvie, Falk, Ferring, Hospira, MSD and Takeda outside the submitted work. $\mathrm{PH}$ : consulting fees, speaking fees and/or honoraria from AbbVie and Pfizer. KR: personal fees from Roche, AbbVie, UCB, BMS and Celgen outside the submitted work. AZ: grants and personal fees from AbbVie, BMS, MSD, Pfizer, Roche and UCB outside the submitted work. JL: personal fees from Sandoz and Pfizer outside the submitted work.

Patient consent Obtained.

Ethics approval The study protocol was approved by the ethics committee of the Charité- Universitätsmedizin Berlin.

Provenance and peer review Not commissioned; externally peer reviewed.

Open Access This is an Open Access article distributed in accordance with the Creative Commons Attribution Non Commercial (CC BY-NC 4.0) license, which permits others to distribute, remix, adapt, build upon this work non-commercially, and license their derivative works on different terms, provided the original work is properly cited and the use is non-commercial. See: http://creativecommons.org/ licenses/by-nc/4.0/

\section{REFERENCES}

1 Hart AR, Kennedy HJ, Stebbings WS, et al. How frequently do large bowel diverticula perforate? An incidence and cross-sectional study. [Article]. Eur J Gastroenterol Hepatol 2000;12:661-5.

2 Morris CR, Harvey IM, Stebbings WSL, et al. Anti-inflammatory drugs, analgesics and the risk of perforated colonic diverticular disease. $\mathrm{Br} J$ Surg 2003:90:1267-72.

3 Svenningsen $\mathrm{P}$, Manoharan T, Foss NB, et al. Increased mortality in the elderly after emergency abdominal surgery. Dan Med J 2014:61:A4876.

4 Prior P, Symmons DP, Scott DL, et al. Cause of death in rheumatoid arthritis. $\mathrm{Br} J$ Rheumatol 1984;23:92-9.

5 Henry DA, Johnston A, Dobson A, et al. Fatal peptic ulcer complications and the use of non-steroidal anti-inflammatory drugs, aspirin, and corticosteroids. $\mathrm{Br} \mathrm{Med} J$ (Clin Res Ed) 1987;295:1227-9.

6 Laine L, Smith R, Min K, et al. Systematic review: the lower gastrointestinal adverse effects of non-steroidal anti-inflammatory drugs. Aliment Pharmacol Ther 2006;24:751-67.
7 Lanas A, Serrano P, Bajador E, et al. Evidence of aspirin use in both upper and lower gastrointestinal perforation. Gastroenterology 1997;112:683-9.

8 Langman MJ, Morgan L, Worrall A. Use of anti-inflammatory drugs by patients admitted with small or large bowel perforations and haemorrhage. $\mathrm{Br}$ Med J (Clin Res Ed) 1985;290:347-9.

9 Curtis JR, Xie F, Chen L, et al. The incidence of gastrointestinal perforations among rheumatoid arthritis patients. Arthritis Rheum 2011;63:346-51.

10 Mpofu S, Mpofu CMA, Hutchinson D, et al. Steroids, non-steroidal antiinflammatory drugs, and sigmoid diverticular abscess perforation in rheumatic conditions. Ann Rheum Dis 2004;63:588-90.

11 Piekarek K, Israelsson LA. Perforated colonic diverticular disease: the importance of NSAIDs, opioids, corticosteroids, and calcium channel blockers. Int J Colorectal Dis 2008:23:1193-7.

12 Závada J, Lunt M, Davies $\mathrm{R}$, et al. The risk of gastrointestinal perforations in patients with rheumatoid arthritis treated with anti-TNF therapy: results from the BSRBR-RA. Ann Rheum Dis 2014;73:252-5.

13 Schiff MH, Kremer JM, Jahreis A, et al. Integrated safety in tocilizumab clinical trials. Arthritis Res Ther 2011;13:1-13.

14 Listing J, Kekow J, Manger B, et al. Mortality in rheumatoid arthritis: the impact of disease activity, treatment with glucocorticoids, TNF $\alpha$ inhibitors and rituximab. Ann Rheum Dis 2015:74:415-21.

15 van Vollenhoven RF, Keystone EC, Furie R, et al. Gastrointestinal safety in patients with rheumatoid arthritis treated with tocilizumab: data from Roche clinical trials. Arthritis Rheum 2009:60:1613.

16 Yamamoto K, Goto H, Hirao K, et al. Longterm safety of tocilizumab: results from 3 years of followup postmarketing surveillance of 5573 patients with rheumatoid arthritis in Japan. J Rheumatol 2015;42:1368-75.

17 Curtis JR, Perez-Gutthann S, Suissa $S$, et al. Tocilizumab in rheumatoid arthritis: A case study of safety evaluations of a large postmarketing data set from multiple data sources. Semin Arthritis Rheum 2015:44:381-8.

18 Myasoedova E, Matteson EL, Talley NJ, et al. Increased incidence and impact of upper and lower gastrointestinal events in patients with rheumatoid arthritis in Olmsted County, Minnesota: a longitudinal population-based study. J Rheumatol 2012;39:1355-62

19 Curtis JR, Lanas A, John A, et al. Factors associated with gastrointestinal perforation in a cohort of patients with rheumatoid arthritis. Arthritis Care Res (Hoboken) 2012:64:1819-28.

20 Siegmund B, Lehr HA, Fantuzzi G. Leptin: a pivotal mediator of intestinal inflammation in mice. Gastroenterology 2002;122:2011-25.

21 Siegmund B, Sennello JA, Jones-Carson J, et al. Leptin receptor expression on T lymphocytes modulates chronic intestinal inflammation in mice. Gut 2004;53:965-72.

22 Peyrin-Biroulet L, Gonzalez F, Dubuquoy L, et al. Mesenteric fat as a source of $\mathrm{C}$ reactive protein and as a target for bacterial translocation in Crohn's disease. Gut 2012;61:78-85

23 Zulian A, Cancello R, Micheletto G, et al. Visceral adipocytes: old actors in obesity and new protagonists in Crohn's disease? Gut 2012;61:86-94. 